Neeraj Sood*, Zachary Wagner, Peter Huckfeldt and Amelia M. Haviland

\title{
Price Shopping in Consumer-Directed Health Plans
}

\begin{abstract}
We use health insurance claims data from 63 large employers to estimate the extent of price shopping for nine common outpatient services in consumer-directed health plans (CDHPs) compared to traditional health plans. The main measures of price shopping include (1) the total price paid on the claim, (2) the share of claims from low- and high-cost providers, and (3) the savings from price shopping relative to choosing prices randomly. All analyses control for individual and zip code level demographics and plan characteristics. We also estimate differences in price shopping within CDHPs depending on expected health care costs and whether the service was bought before or after reaching the deductible. For eight out of nine services analyzed, prices paid by CDHP and traditional plan enrollees did not differ significantly; CDHP enrollees paid 2.3\% less for office visits. Similarly, office visits was the only service where CDHP enrollment resulted in a significantly larger share of claims from low-cost providers and greater savings from price shopping relative to traditional plans. There was also no evidence that, within CDHP plans, consumers with lower expected medical expenses exhibited more price shopping or that consumers exhibited more price shopping before reaching the deductible.
\end{abstract}

Keywords: consumer directed health plans; high deductible health plans; price

\footnotetext{
*Corresponding author: Neeraj Sood, University of Southern California, e-mail: nsood@healthpolicy.usc.edu

Zachary Wagner: Leonard D. Schaeffer Center for Health Policy and Economics

Peter Huckfeldt and Amelia M. Haviland: Carnegie Mellon University, RAND Corporation
}

\section{Introduction}

"Consumer-directed" health plans (CDHPs), characterized by high deductibles and tax-advantaged personal health accounts, are becoming increasingly popular. Enrollment in these plans has risen from 4\% to $17 \%$ of employer-provided insurance between 2006 and 2010, with 59\% of large employers (with $1000+$ employees) offering such a plan in 2011 (Kaiser Family Foundation and 
HRET 2011; Towers Watson and National Business Group on Health 2012). In exchange for the high deductible, CDHP enrollees typically face lower monthly premiums. To help cover the high deductible, CDHP enrollees, their employers, or both can contribute to tax-free personal health accounts. The goal of CDHPs is to reduce health care costs while maintaining health care quality through the use of higher value services. Proponents of CDHPs argue that by having enrollees pay the entire price out of pocket for services received before reaching the deductible, enrollees have increased financial incentive to "shop" for lower price providers of health care. Moreover, many CDHPs aim to facilitate price shopping by providing online decision support tools. To the extent that enrollees respond to these incentives, CDHPs could lower health care costs and even stimulate price competition among providers.

Prior research demonstrates that CDHPs reduce short-term costs (Lo Sasso et al. 2004; Parente et al. 2004; Feldman and Parente 2010; Buntin et al. 2011). However, it remains unclear whether these reductions reflect price shopping or indiscriminate reductions on the part of enrollees. Numerous papers have shown short-term reductions in emergency department use and hospitalization rates after CDHP enrollment (Wharam et al. 2007, 2011b; Haviland et al. 2011), potentially indicating more efficient use of health care. However, the prior literature shows mixed effects on use of high-value services. Some papers show decreased use of some preventive services (Wharam et al. 2008, 2011a; Buntin et al. 2011) and reductions in both high-and low-priority office visits (Hibbard et al. 2008). In contrast, research examining a single CDHP shows no evidence of reductions in preventive care use relative to traditional plans (Rowe et al. 2008). Estimated impacts on pharmaceutical utilization are similarly mixed: results from a single employer show discontinuation of drugs treating asymptomatic chronic conditions and little change in use of generics (Greene et al. 2008), while estimates across employers and plans show increased use of generic drugs (Haviland et al. 2011). Other research finds that individuals in CDHPs with chronic conditions are more likely to delay care (Galbraith et al. 2012), and CDHPs restrict access to care and increase financial burden for sicker or lower-income individuals (Davis et al. 2005; Galbraith et al. 2011). There is no research to date investigating whether CDHP enrollees shop for lower cost care.

Even with the right incentives, price shopping for health care can be challenging, as consumers often do not have information on the prices charged by different providers. Although a number of states have implemented initiatives aimed at improving price transparency, the effects of such policies remain largely unknown (Sinaiko and Rosenthal 2011). Early evidence shows that one initiative in New Hampshire had little impact on price variation ( $\mathrm{Tu}$ and Lauer 2009). As most of these initiatives do not make available to patients the price they will 
actually be exposed to - the one negotiated by their health insurance plan (focusing instead on charges or averages over all negotiated prices) - challenges are likely to remain. Several CDHPs attempt to address these concerns and facilitate price shopping by providing online decision support tools with information on prices charged by different providers in their network.

This study is the first to investigate whether CDHPs are effective in encouraging enrollees to shop for lower-cost providers of health care. We examine the prices paid for common outpatient procedures using claims data from 63 large employers more than half of which offered CDHPs in 2007. In order to identify the role played by price transparency, we investigate a range of common outpatient services with both higher and lower levels of price transparency and scope for price shopping (e.g., office visit vs. pelvic ultrasound).

\section{Methods and Data}

\subsection{Study Design}

The objective of our analyses is to estimate differences in price-shopping behavior between patients in CDHPs and patients in conventional health plans. We define a CDHP plan as including a deductible of $\$ 1000$ or more for individual coverage (twice this for family coverage). CDHP plans typically are defined to include a tax-advantaged health savings account (employee owned, employer contribution not required) or health reimbursement arrangement (employer owned, employer contribution required); 91\% of CDHP plans in our sample include such personal health accounts that comprise 94\% of all CDHP members in our sample. We focus on total prices for the following nine common outpatient procedures and services: arthrocentesis, chest X-ray, colonoscopy, emergency department visit, flu vaccine, mammogram, office visit, pelvic ultrasound, and preventive visits.

We first estimate differences in total prices paid by CDHP beneficiaries relative to prices paid by enrollees in traditional plans. If CDHP patients are more price sensitive, they may be more likely to seek lower-cost providers of procedures and services. However, unadjusted differences in prices may also reflect differences in individual characteristics related to the propensity to price shop or differences in health plans affecting bargaining power to negotiate lower prices for procedures and services. Reflecting this, we also compute adjusted differences, controlling for individual and zip code level demographic characteristics and plan characteristics. 
In further analysis, we more explicitly account for differential price availability between CDHP and conventional plans. Specifically, the set of available prices for a given service may be higher for enrollees in CDHPs compared to conventional plans if the CDHP has a smaller share of the market and thus less bargaining power when negotiating with providers.

In such a case, CDHP enrollees may pay higher prices than conventional plan enrollees even if CDHP enrollees price shop more than enrollees in conventional plans. To account for this, we identify the distribution of unique prices paid for each service within a plan and metropolitan statistical area (MSA). If CDHP enrollees are more likely to price shop than enrollees in conventional plans, we would expect a higher proportion of CDHP claims to come from providers in the lower end of the distribution of offered prices in comparison to this proportion in traditional plans.

However, it is possible that the proportion of enrollees purchasing health care from the lower end of the observed distribution of offered prices is sensitive to the distribution of unique prices. Therefore, the results from the above analysis might be biased if there are large differences in the distribution of unique price points across CDHP and conventional plans. Table 1 shows that although there are more unique price points in traditional plans, there is no systematic relationship between plan type (CDHP vs. conventional) and the standard deviation (SD) or interquartile range of the distribution of unique price points. However, there could still exist differences in access to specific price points between CDHPs and conventional plans. For example, conventional plans might have larger networks and thus more providers with low price points compared to early CDHPs from smaller carriers, biasing our estimates against observing price-shopping behavior. To account for such differences, we examine within-plan changes in price-shopping behavior for CDHP enrollees before and after reaching the deductible. CDHP patients have a greater incentive to price shop prior to reaching the deductible when they must pay the full cost of nonexempt services. If CDHP enrollees exhibit price-shopping behavior, we would expect to see a higher proportion of claims for providers in the lower end of the price distribution prior to reaching the deductible relative to after reaching the deductible.

However, one potential problem with the above analysis is that if enrollees are forward-looking then their price-seeking behavior might be determined by the end-of-year price of health care services rather than the current price (Aron-Dine et al. 2012). If this is the case, we would expect less price shopping from enrollees more likely to reach the deductible as they face a lower end-of-year price. We examine this by estimating whether CDHP enrollees who are more likely to reach the deductible are less likely to demonstrate price-shopping behavior. We note that behaviorally, this requires patients to have no "myopia;" that is, they are impervious to seeing and paying out of pocket the entire price of care prior to meeting their deductible, which, while rational, may be unlikely. 
Table 1: Descriptive Statistics of Plan Price Points.

\begin{tabular}{|c|c|c|c|c|c|c|}
\hline \multirow[t]{2}{*}{ Procedure } & \multicolumn{2}{|c|}{$\begin{array}{r}\text { Average Number } \\
\text { of Price Points per } \\
\text { Plan-MSA }\end{array}$} & \multicolumn{2}{|r|}{ Average SD } & \multicolumn{2}{|c|}{ Average Q1, Q3 } \\
\hline & CDHP & Traditional & CDHP & Traditional & CDHP & Traditional \\
\hline Arthrocentesis & 10 & 15 & 28.69 & 36.02 & $(67,103)$ & $(63,107)$ \\
\hline Chest X-ray & 19 & 24 & 25.26 & 30.67 & $(22,54)$ & $(22,62)$ \\
\hline Colonoscopy & 10 & 14 & 254.27 & 226.85 & $(358,6450)$ & $(349,642)$ \\
\hline Emergency department visit & 17 & 25 & 87.88 & 92.69 & $(133,254)$ & $(115,245)$ \\
\hline Flu vaccine & 9 & 13 & 3.01 & 2.92 & $(13,17)$ & $(13,17)$ \\
\hline Mammogram & 16 & 20 & 31.56 & 32.53 & $(57,102)$ & $(53,101)$ \\
\hline Office visit & 27 & 46 & 11.97 & 12.14 & $(57,73)$ & $(57,72)$ \\
\hline Pelvic ultrasound & 14 & 17 & 50.85 & 59.77 & $(67,137)$ & $(66,146)$ \\
\hline Preventive visit & 16 & 20 & 20.07 & 20.96 & $(115,143)$ & $(114,142)$ \\
\hline
\end{tabular}

The scope for individuals to price shop varies across services and may be a function of price transparency, the urgency of the service, and whether patients have agency to choose providers. For services such as office visits, prices are knowable and relatively transparent, especially as the service is used repeatedly on a regular basis. Patients may have time to search for the lowest cost provider, and patients have agency to choose from a range of providers. However, some consumers might find it difficult to change primary care providers even if they are expensive, especially if they have a long-standing relationship with that provider. In contrast, even with transparent pricing, there is little scope for patients to price shop for the lowest-cost emergency department after an accident. However, patients might have the ability to price shop for nonurgent emergency room care. For services such as pelvic ultrasound or chest X-ray, there may be less ability to choose a lower-cost provider as the service might be bundled with a physician visit, or a patient's physician may always use a particular facility for such services. Moreover, price shopping for laboratory tests might be easier, as the quality of services offered is likely to be similar across providers. Reflecting this potential heterogeneity, we examine price shopping for a range of outpatient procedures and services.

\subsection{Data Sources and Study Population}

The study population is drawn from full-time employees and their dependents working for 63 employers in 2007. Roughly half of the employers were recruited 
on the basis of their offering of a CDHP between 2003 and 2007, representing a range of geographic locations, size, and industries. The other half was drawn from the Thomson Reuters MarketScan database to roughly match the characteristics of the CDHP-offering firms. To account for variation in prices by geographic location, we define each plan unit as the unique combination of plan by MSA, meaning that a plan that spans across two MSAs is considered two distinct plan units. Henceforth, we will use the term "plan-MSA" for these units.

The base sample includes beneficiaries incurring a claim for one of the nine outpatient services in the analysis. We identify these services in the claims using the designated Current Procedure Technology (CPT) code. Each service has a number of variations, all identified by a unique CPT code; we use the most common CPT code for each procedure. The list of services, their CPT codes, and a detailed description are displayed in Appendix Table A1.

We limit the sample to include only plan-MSAs with at least 50 claims for a given service in 2007. Our empirical strategy requires within-plan-MSA variation in prices; thus, we only include plan-MSA combinations with at least three unique price points for a particular service. As shown in Table 2, the final sample includes 6.3 million claims from 2.1 million enrollees in 9377 plan-MSA combinations ( $21 \%$ of which are CDHPs).

Table 2: Descriptive Statistics for CDHP and Traditional Plans Included in Sample.

\begin{tabular}{|c|c|c|}
\hline & CDHP & Traditional \\
\hline Number of plans ${ }^{a}$ & 1953 & 7424 \\
\hline Number of enrollees & 245,176 & $1,907,973$ \\
\hline Number of claims & 631,434 & $5,640,554$ \\
\hline Average number of enrollees per plan-MSA & $15,545^{\star \star \star}$ & 35,770 \\
\hline Age of enrollee & $30^{\star \star \star *}$ & 32 \\
\hline Male & 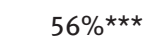 & $54 \%$ \\
\hline Median household income & $\$ 56,817^{\star \star}$ & $\$ 52,471$ \\
\hline$\%$ Unemployed & $4.1 \%$ *** & 4.7 \\
\hline$\%$ College degree & 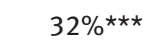 & 27 \\
\hline$\%$ High school degree & 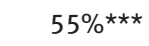 & 57 \\
\hline$\%$ Hispanic & 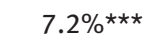 & 7.1 \\
\hline$\%$ Black & 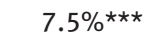 & 9.9 \\
\hline$\%$ White & $80 \% * * *$ & 78 \\
\hline Average number of enrollees & $15,545^{\star \star \star}$ & 35,770 \\
\hline
\end{tabular}

$\star \star \star x<0.001$.

aplans are defined as unique by plan and MSA.

Note: $t$-tests are used to test for significant differences. 


\subsection{Study Variables}

\subsubsection{Outcomes}

The primary outcome in the analyses is the total amount paid for a particular claim. The total amount includes payments by both the insurer and the enrollee. To account for errors and outliers, we drop claims that have negative payment values and claims with payments that are greater than the 99th percentile or less than the 1st percentile of payments for each respective service, resulting in $1.8 \%$ of claims being dropped. To ensure the validity of claims and the distribution of unique price points, we require that the payment on a claim be observed more than once within a service and plan-MSA; otherwise, the claim is dropped ( $5 \%$ of claims were dropped as a result of this restriction).

For analyses investigating the distribution of claims over unique price points, we construct variables indicating whether the claim was from a provider in the highest or lowest tercile of a plan-MSA's distribution of unique prices for each service. Specifically, we construct the set of unique prices for a particular service within each plan-MSA combination, define terciles in the unique price distribution, and then indicate claims from the highest tercile as coming from "high-cost" providers and indicate claims from the lowest tercile as coming from "low-cost" providers.

Finally, we construct a variable, "Savings from Price Shopping," indicating the savings due to price shopping relative to costs if patients randomly chose a price from the available price points for a particular service. This outcome is defined in Equation (1):

$$
\text { Savings from price shopping }_{i p}=1-\frac{P_{i p}}{\frac{1}{N_{p}} \sum_{u=1}^{N_{p}} P_{u p}}
$$

where $P_{i p}$ represents the price on claim $i$ from plan-MSA $p, P_{u p}$ represents the $u$ th unique price point for plan-MSA $p$, and $N_{p}$ represents the number of unique price points in plan-MSA $p$.

\subsubsection{Explanatory Variables}

The explanatory variable of interest is whether a claim belongs to a CDHP or traditional plan enrollee, whether a CDHP enrollee has reached their deductible, or the CDHP enrollee's prospective risk of reaching the deductible. 
Individual-level demographic controls include age, age squared, indicators for being $<2$ years old and between 2 and 16 years old, and gender. Zip code level socioeconomic status controls include the share of residents that are Hispanic, Black, and White; the share of residents that have a high school and college education; the unemployment rate; and median household income. Plan-level controls include the number of claims for each service and total number of enrollees to serve as proxies for bargaining power, and coinsurance rate and average copayment to control for cost-sharing features of each plan's benefit design.

\subsection{Statistical Analysis}

We complete three analyses for each of the nine services, each estimated at the claim level. First, we regress the natural log of total payment on just an indicator for CDHP enrollment to estimate the percent difference in mean prices between CDHPs and conventional plans using ordinary least squares. We then estimate adjusted percent differences in prices paid controlling for the individual and plan characteristics described above.

Second, we estimate probit regression models where the outcome is either that the claim came from a low-cost provider (a provider with a price in the lowest tercile of the unique prices for a plan-MSA) or a high-cost provider (a provider with a price in the highest tercile of the unique prices distribution for a planMSA). The explanatory variables include the CDHP enrollment indicator and the individual and plan characteristics described above. We then estimate linear regressions of the savings variable [defined in Equation (1)] on the CDHP indicator, and controls.

Next, for CDHP enrollees only, we run probit regressions on the low- and high-cost indicators with the "predeductible" indicator, demographic characteristics, plan-level characteristics, and a cubic specification of calendar month to control for seasonal variation in utilization as predictors. We report the adjusted shares of pre- and postdeductible claims that fall into each category (using recycled predictions) and the difference in these shares pre- and postdeductible.

We then use linear regression models to model average savings among CDHP enrollees from price shopping pre- vs. postdeductible including controls for individual and plan characteristics and a cubic in calendar month.

It is possible that CDHP enrollees are forward-looking and anticipate reaching their deductible, in which case price-seeking behavior would not change after reaching the deductible (enrollees would be paying up to the deductible out of pocket regardless) (Aron-Dine et al. 2012). To account for this, we perform an additional analysis using the 2006 prospective risk score; this score predicts 
health care costs in 2007 based on health utilization and demographic information from 2006. A higher 2006 prospective risk score indicates a greater likeliness of reaching the deductible in 2007, and therefore less incentive to price shop. For the sample of CDHP enrollees, we regress the natural log of price paid for a particular service on the prospective risk score (in the main analysis, the risk score is defined as a continuous variable; in alternative specifications we used the quintile of risk score), controlling for individual, and plan characteristics and a cubic polynomial in calendar month.

We cluster standard errors at the plan-MSA level for all analyses. Obtaining separate estimates for the nine services in our sample poses a potential multiple-comparisons problem. We account for this by implementing the Bonferroni correction in our statistical inference, multiplying each $p$ value by nine. All analyses were performed using STATA/MP 12.0 (StataCorp LP, TX, USA) and the study was approved by the institutional review boards of each of the study authors' institutions.

\section{Results}

\subsection{Study Population}

Table 2 displays the summary statistics for enrollees with claims for the nine services in our sample. The statistical significance of the difference between CDHP and traditional plans is indicated for average measures. CDHP plans comprise roughly $21 \%$ of the sample (1953 plans compared to 7424 traditional plans) and approximately $10 \%$ of claims. CDHP plans have fewer enrollees. CDHP enrollees with claims for common outpatient services are younger, more likely to be female, and live in zip codes with higher income and education levels ( $p<0.01$ for all). CDHP plans have a median individual deductible of $\$ 1250$ with first and third quartiles of $\$ 1100$ and $\$ 1750$, respectively. Traditional plans have substantially lower deductibles with a median of $\$ 250$ and first and third quartiles of $\$ 0$ and $\$ 400$, respectively.

\subsection{Price Differences in CDHPs Relative to Traditional Plans}

Table 3 shows the mean price paid for each service in CDHPs and traditional plans for all nine procedures along with the 25th and 75th percentiles (across all CDHP or traditional claims) and the percentage differences with and without adjustment for 





controls. The unadjusted differences are either statistically insignificant or show that CDHP patients pay more for services than non-CDHP patients (emergency department visits, $p<0.01$ ). The 25 th and 75 th percentiles are similar between CDHP and traditional plans and for most services imply a wide range of available prices (and thus scope for price shopping). Flu vaccines have somewhat narrower ranges indicating a smaller scope for price shopping for these services. Once demographic and plan-level controls are included in column 4, most point estimates on the percent differences are reduced and remain statistically insignificant except that after adjustment the price of an office visit is 2.3\% less for CDHP enrollees compared to the price of an office visit for traditional plan enrollees $(p<0.01)$.

\subsection{Price Selection in CDHPs Relative to Traditional Plans}

Table 1 shows the average number of price points per plan, the average SD, and the average first and third quartiles for CDHP and traditional plans. This table shows that there are more price points in traditional plans; however, there is no systematic relationship between plan type (CDHP vs. traditional) and the SD or interquartile range of the distribution of price points.

The first two panels of Table 4 display estimates from probit regressions, showing the predicted share of CDHP and traditional plan claims from low- and high-cost providers. The table also reports the difference in these shares between CDHP and traditional plans. Across the nine services in the sample, there is only a statistically significant difference in the share of low-cost providers for office visits. The share of claims of low-cost providers in CDHP plans is 6.4\% points higher than the share of claims of low-cost providers in traditional plans $(p<0.01)$. The only statistically significant difference in the share of high-cost providers is for colonoscopies. The share of claims of high-cost providers in CDHP plans is $28.8 \%$ points higher than the share of claims from high-cost providers in traditional plans $(p<0.05)$. Finally, to test the robustness of our results to our tercilebased specification of high- and low-cost providers, we run an additional analysis using within-plan price point percentile (continuous) as the dependent variable and find that our results are consistent with the main analysis. In particular, CDHP enrollment has a negative and significant coefficient for office visits only.

The third panel of Table 4 shows estimates of savings from price shopping for CDHP and traditional plans. This measure indicates the average percent difference in observed prices (for either CDHP or traditional plans) relative to what would be seen if patients randomly choose providers from the observed unique price points; a positive percentage indicates positive savings and evidence of price shopping. Panel 3 shows price-shopping-related savings for CDHPs for 


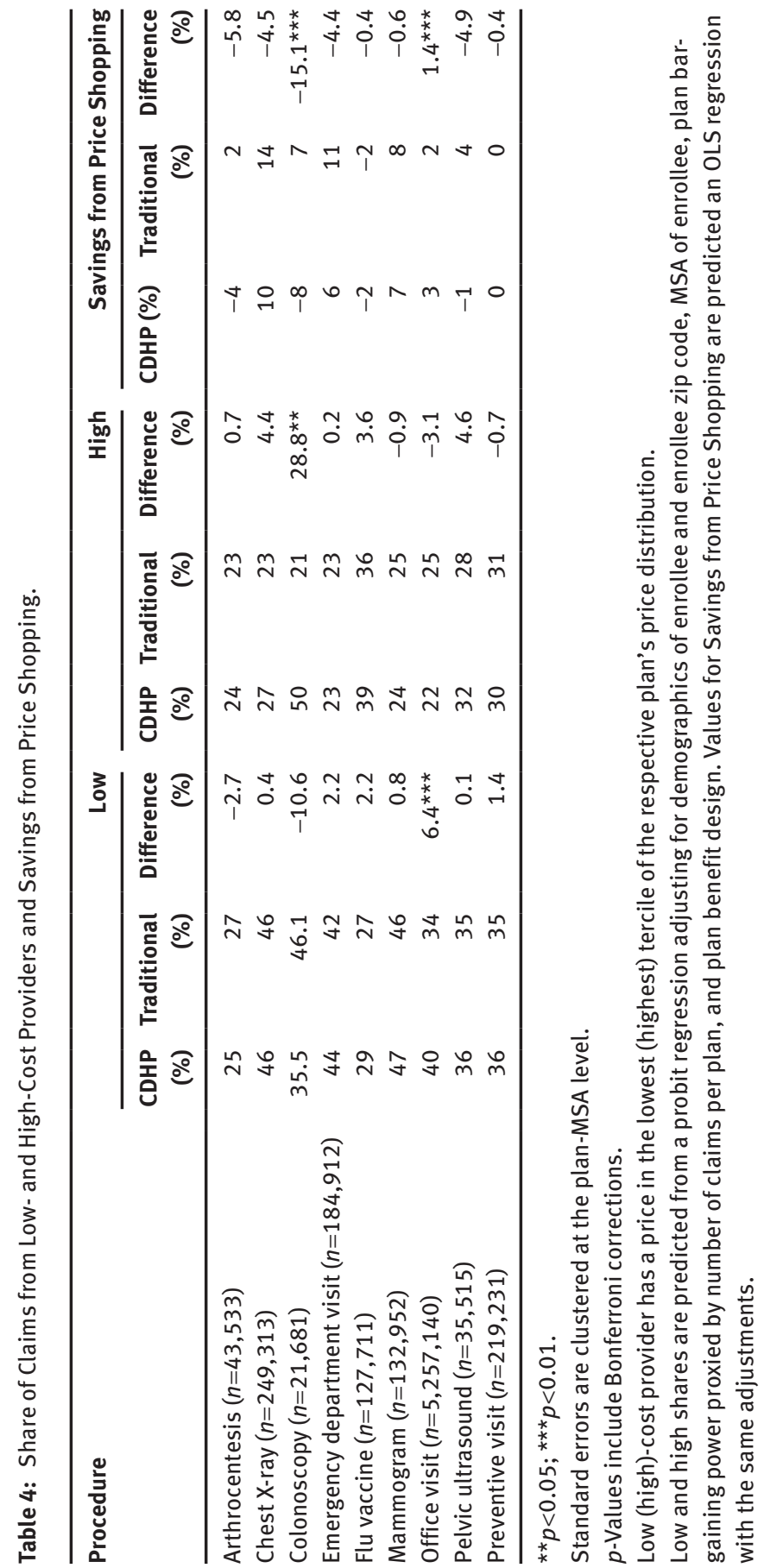


only five procedures, whereas we see price-shopping-related savings for eight of the nine procedures for traditional plans. Savings from price shopping are only greater for CDHP plans compared to traditional plans for office visits $(p<0.01)$. Claims for colonoscopies show a greater degree of price shopping in traditional plans compared to CDHP plans $(p<0.01)$.

\subsection{Price Selection Pre- vs. Postdeductible}

The first and second panels of Table 5 show differences in the predicted share of claims from high- and low-cost providers, pre- and postdeductible. If CDHP enrollees are price sensitive and engage in price shopping, we would expect a higher fraction of claims from low-cost providers prior to reaching the deductible relative to after the deductible when patient cost sharing is lower. Similarly, we would expect a lower fraction of claims for high-cost providers prior to reaching the deductible relative to after reaching the deductible. However, panel 1 shows that predicted shares for low-cost providers are similar before and after reaching the deductible $(p>0.1)$ for six of the nine services. The only procedure where the share of low-cost providers is significantly higher prior to reaching the deductible is preventive care visits $(p<0.01)$. For chest X-rays and colonoscopies the low-cost provider share is significantly lower prior to reaching the deductible $(p<0.01)$. Panel 2 shows that the fraction of claims for high-cost providers are similar preand postdeductible for all services $(p>0.1)$.

The third panel shows whether the savings from price shopping change after reaching the deductible. We would expect greater savings prior to the deductible relative to after reaching the deductible, as cost sharing decreases. However, savings are similar in both periods for all services $(p>0.1)$ except for chest X-ray and pelvic ultrasound, which implies 5.7\% $(p<0.01)$ and 7.7\% $(p<0.1)$ more savings from price shopping after reaching the deductible $(p<0.01)$, respectively.

Finally, we find no relationship between CDHP enrollees' prospective risk score and price paid for all procedures (results not presented). This result is consistent with a lack of forward-looking behavior, as patients who anticipated spending past the deductible would have reduced incentive to price shop.

\section{Discussion}

Overall, we find that prices for most common outpatient services are indistinguishable in CDHPs and traditional plans. The only exception to these null find- 


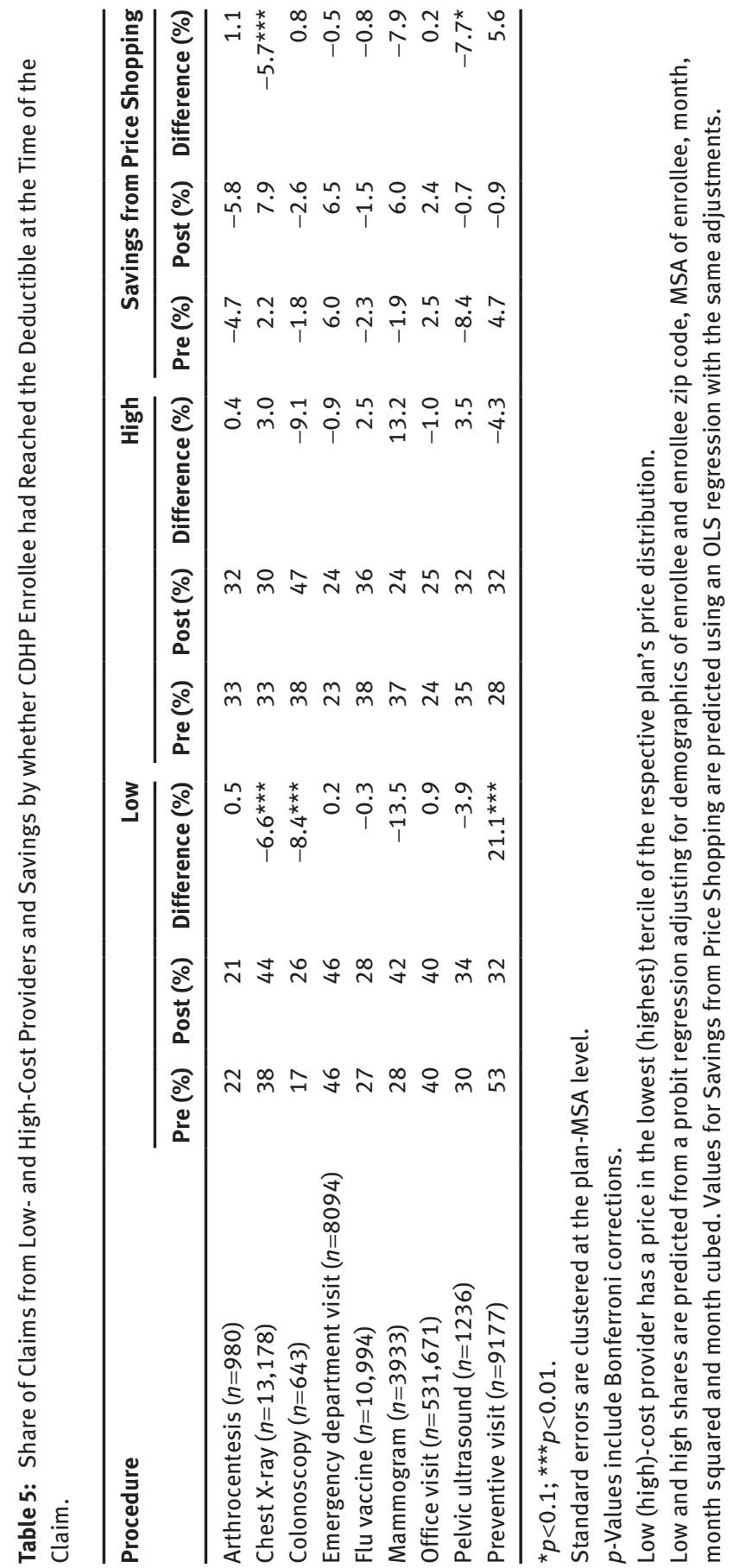


ings is office visits which fall at the upper end of the price transparency and price shopping scope continuum. The lower average prices, greater use of low-cost providers, and greater plan-level savings due to price shopping for office visits in CDHPs relative to traditional plans suggest that CDHPs may be effective at incentivizing price shopping for office visits. However, the magnitude of the effect is modest.

Consistent with the above null findings, comparing price shopping by CDHP enrollees, we see no evidence of greater price shopping for CDHP enrollees prior to meeting their deductible compared with after meeting their deductible. One possible explanation for this lack of change is that patients are returning to the same providers they used prior to meeting their deductible and another is that patients are forward-looking and thus not sensitive to this within-year change in the price.

The findings of this study should be viewed in light of its limitations. First, whereas we address potential differential bargaining power of CDHPs vs. traditional plans by analyzing share of high- vs. low-cost providers across CDHP and traditional plans and within CDHPs before and after reaching the deductible, bargaining power could still play a role. For example, traditional plans may be able to negotiate the same low price point for a larger share of their providers relative to CDHP plans. Also, a traditional plan may have larger networks and thus many providers offering the same (relative) low price point. This would make low price providers the default option for many traditional plan enrollees, whereas CDHP enrollees may have a more difficult time finding prices at the lower end of the distribution.

Second, we only look at price outcomes, but not quality. Thus, CDHP enrollees may make "value"-based decisions using quality outcomes that we do not observe. Ideally, we would like data on the provider choices for each consumer including data on the prices charged by each provider, the quality of care for each provider, and information on amenities and other provider characteristics that might influence such choices. Such data could be used to estimate choice models to understand how the role of price and quality in determining provider choice differs across CDHP and traditional plans. However, to the best of our knowledge such detailed data on providers is not available.

Finally, our study relies on observational data, and thus CDHP status is not randomly assigned. Although we control for a range of observable characteristics, there may still be selection on unobservable characteristics biasing the results. Specifically, our estimates of the effects of CDHPs on price shopping might be positively biased if individuals with an inherent propensity to price shop (unobserved to the researcher) are more likely to enroll in CDHP plans. If this is the case, our findings might overstate the effects of CDHPs on price shopping. Moreover, 
the CDHP enrollees utilizing outpatient care in this study are healthier, lower utilizers of health care and somewhat higher income than those in traditional plans. Thus, CDHP enrollees might be less experienced health care consumers and less sensitive to prices. If this is the case, our findings might be understating the effects of CDHPs on price shopping.

Overall, our results suggest that CDHPs do not stimulate "price shopping” for most common outpatient services. This naturally raises the question: Why do the incentives of CDHPs appear to be largely ineffective in motivating price shopping for outpatient services? There are a number of potential factors. In particular, there may be limited scope for patients to search for low-cost providers. Other studies have shown that the price transparency of health services is low, and early state-level efforts to improve price transparency have so far shown little effect. This is compounded by uncertainty in the degree to which patients perceive their agency to choose alternative providers for some services.

Similarly, it may be that price shopping is not an immediate response to enrolling in a CDHP plan, perhaps because patients do not perceive the degree of price variation. One key factor encouraging price shopping may be the availability of clear signals of price (such as generic drugs, or "outof-network" providers). Another important determinant of price shopping may be repeated use of a service. The only service where we found limited evidence of price shopping was for outpatient doctor visits, which occur with some regularity and thus offer opportunities to "learn" to price shop after having to pay for a service that may then outweigh patients' potential attachment to the providers seen most often. Finally, consumers might not have the agency to shop for some diagnostic services if the service is bundled with a physician visit, or a patient's physician may always use a particular facility for such services.

Improved education for enrollees about benefit design and more aggressive, health insurance carrier specific, price and quality transparency initiatives may be the next steps for employers, health plans, and policy makers to increase consumerism in health care decision-making.

Acknowledgment: Funding was provided by the California Health Care Foundation and National Institute for Aging grant R01 AG043850-01. 







\section{References}

Aron-Dine, A., L. Einav, A. Finkelstein and M. R. Cullen (2012) Moral Hazard in Health Insurance: How Important Is Forward Looking Behavior? Cambridge, MA: National Bureau of Economic Research.

Buntin, M. B., A. Haviland, R. McDevitt and N. Sood (2011) "Health Care Spending and Preventive Care in High-Deductible and Consumer-Directed Health Plans," American Journal of Managed Care, 17(3):222-230.

Davis, K., M. Doty and A. Ho (2005) How High Is Too High? Implications of High-Deductible Health Plans. The Commonwealth Fund, Pub No. 816.

Feldman, R. D. and S. T. Parente (2010) "Enrollee Incentives in Consumer-Directed Health Plans: Spend Now or Save Later?” Forum for Health Economics and Policy, 13(2):Article 3.

Galbraith, A. A., D. Ross-Degnan, S. B. Soumerai, M. B. Rosenthal, C. Gay and T. A. Lieu (2011) "Nearly Half of Families in High-Deductible Health Plans Whose Members Have Chronic Conditions Face Substantial Financial Burden," Health Affairs, 30(2):322-331.

Galbraith, A. A., S. B. Soumerai, D. Ross-Degnan, M. B. Rosenthal, C. Gay and T. A. Lieu (2012) "Delayed and Forgone Care for Families with Chronic Conditions in High-Deductible Health Plans," Journal of General Internal Medicine, 27:1105-1111.

Greene, J., J. Hibbard, J. F. Murray, S. M. Teutsch and M. L. Berger (2008) "The Impact of Consumer Directed Health Plans on Prescription Drug Use," Health Affairs, 27(4):1111-1119.

Haviland, A. M., R. D. McDevitt, N. Sood and M. S. Marquis (2011) "The Effects of ConsumerDirected Health Plans on Episodes of Health Care," Forum for Health Economics and Policy, 14(2):Article 9.

Hibbard, J. H., J. Greene and M. Tusler (2008) “Does Enrollment in a CDHP Stimulate Cost-Effective Utilization?" Medical Care Research and Review, 65(437):437-449.

Kaiser Family Foundation and HRET (2011) Survey of Employer-Sponsored Health Benefits 1999-2011.

Lo Sasso, A., T. Rice, J. Gabel and H. Whitmore (2004) "Tales from the New Frontier: Pioneers' Experiences with Consumer-Driven Health Care," Health Services Research, 39(4p2): 1071-1090.

Parente, S. T., R. Feldman and J. B. Christianson (2004) "Evaluation of the Effect of a ConsumerDirected Health Plan on Medical Care Expenditures and Utilization," Health Services Research, 39(4, Part II):1189-1209.

Rowe, J., T. B. Stevenson, R. Downey and J. Newhouse (2008) "Effect of Consumer-Directed Health Plans on the Use of Preventive and Chronic Illness Services," Health Affairs, 27(1):113-120.

Sinaiko, A. D. and M. B. Rosenthal (2011) "Increased Price Transparency in Health Care Challenges and Potential Effects," New England Journal of Medicine, 364(10):891-892.

Towers Watson and National Business Group on Health (2012) Performance in an Era of Uncertainty: Employer Survey on Purchasing Value in Health Care. Los Angeles, California: Towers Watson.

Tu, H. T. and J. R. Lauer (2009) Impact of Health Care Transparency on Price Variation: The New Hampshire Experience. Washington, DC: Center for Studying Health System Change, Issue Brief No. 128.

Wharam, J. F., B. E. Landon, A. A. Galbraith, K. P. Kleinman, S. B. Soumeraj and D. Ross-Degnan (2007) "Emergency Department Use and Subsequent Hospitalizations Among Members of a High-Deductible Plan,” Journal of The American Medical Association 297(10):1093-1102. 
Wharam, J. F., A. A. Galbraith, K. P. Kleinman, S. B. Soumerai, D. Ross-Degnan and B. E. Landon

(2008) "Cancer Screening Before and After Switching to a High-Deductible Health Plan," Annals of Internal Medicine, 148(9):647-655.

Wharam, J. F., A. J. Graves, B. E. Landon, F. Zhang, S. B. Soumerai and D. Ross-Degnan (2011a)

"Two-Year Trends in Colorectal Cancer Screening After Switch to a High-Deductible Health Plan," Medical Care, 49(9):865-871.

Wharam, J. F., B. E. Landon, F. Zhang, S. B. Soumerai and D. Ross-Degnan (2011b)

"High-Deductible Insurance: Two-Year Emergency Department and Hospital Use,"

American Journal of Managed Care 17(10):e410-e418. 